\title{
Structural Organization of the Corpus Callosum Predicts the Extent and Impact of Cortical Activity in the Nondominant Hemisphere
}

\author{
Mary Colvin Putnam, Gagan S. Wig, Scott T. Grafton, William M. Kelley, and Michael S. Gazzaniga \\ Department of Psychological and Brain Sciences, Center for Cognitive Neuroscience, Dartmouth College, Hanover, New Hampshire 03755
}

\begin{abstract}
Diffusion tensor imaging (DTI) and functional magnetic resonance imaging (fMRI) were combined to examine the relationship between callosal organization and cortical activity across hemispheres. Healthy young adults performed an incidental verbal encoding task (semantic judgments on words) while undergoing fMRI. Consistent with previous studies, the verbal encoding task was associated with left-lateralized activity in the inferior prefrontal cortex (LIPFC). When subjects were divided into two groups based on fractional anisotropy (FA) values in the anterior corpus callosum (DTI), individuals with low anterior callosal FA were found to exhibit greater activity in a homologous region within the right inferior prefrontal cortex (RIPFC) relative to those with high anterior callosal FA. Interestingly, whereas the magnitude of RIPFC activity did not negatively impact subsequent verbal memory performance for individuals with low anterior callosal FA, greater RIPFC activity during verbal encoding was associated with poorer subsequent memory performance for individuals with high anterior callosal FA. Together, these findings provide novel evidence that individual differences in callosal organization are related to the extent of nondominant cortical activity during performance during a lateralized task, and further, that this relationship has consequences on behavior.
\end{abstract}

Key words: corpus callosum; laterality; hemispheric asymmetry; memory; individual differences; neuroimaging

\section{Introduction}

Research with complete callosotomy ("split-brain”) patients has clearly demonstrated that the absence of the corpus callosum prevents the integration of lateralized cognitive functions (Gazzaniga, 2000). Yet, the relationship between the structural organization of the corpus callosum and lateralized cortical processing in the intact brain remains relatively unknown. Early studies reported an inverse relationship between callosal size and cortical asymmetry, particularly in men (Witelson and Goldsmith, 1991; Aboitiz et al., 1992a). More recently, studies have demonstrated that the strength of hemispheric asymmetries declines in normal aging (for review, see Cabeza, 2002), and that this process may be associated with callosal degeneration (Persson et al., 2006). Together, this research suggests that the structural organization of the corpus callosum may contribute to the distribution of pro-

\footnotetext{
Received May 18, 2007; revised Dec. 12, 2007; accepted Jan. 7, 2008.

This work was supported by the Dartmouth Brain Imaging Center and National Institutes of Health Grants R01NS031443-10A2 and P50 NS17778 to M.S.G. M.C.P. was funded by a graduate research fellowship from the National Science Foundation. We thank Beatrice Hahn, Souheil Inati, Tammy Laroche, and Jeff Woodward for their help with data acquisition and analysis, and Margaret Funnell and Souheil Inati for their helpful comments on previous versions of this manuscript.

Correspondence should be addressed to Dr. Mary Colvin Putnam at her present address, Department of Psychiatry, Massachusetts General Hospital, Harvard Medical School, 1 Bowdoin Square, 7th Floor, Boston, MA 02114. E-mail:mputnam1@partners.org.

G. S. Wig's present address: Harvard University, Cambridge, MA 02138.

S. T. Grafton's and M. S. Gazzaniga's present address: Center for Mind and Brain, University of California, Santa Barbara, Santa Barbara, CA 93106.

DOI:10.1523/JNEUROSCI.2295-07.2008

Copyright $\odot 2008$ Society for Neuroscience $\quad$ 0270-6474/08/282912-07\$15.00/0
}

cessing resources between the hemispheres. The current study combines both diffusion tensor imaging (DTI) and functional magnetic resonance imaging (fMRI) to compare both the extent of nondominant cortical activity during performance of a lateralized task in healthy young adults with high and low relative callosal organization [defined as per fractional anisotropy (FA) values] and the impact of this nondominant cortical activity on task performance.

To assess the relationship between callosal organization (FA) and nondominant cortical activity, we took advantage of the well documented material-specific hemispheric asymmetry for memory encoding (Kelley et al., 1998; Wagner et al., 1998a; Wig et al., 2004). Healthy young adults performed an incidental verbal encoding task while undergoing fMRI. In this population, activity in the left inferior prefrontal cortex (LIPFC) has been consistently observed during encoding of verbal material (Kelley et al., 1998); furthermore, LIPFC activity selectively predicts subsequent verbal memory (Wagner et al., 1998b). In contrast, healthy older adults performing verbal encoding tasks often demonstrate LIPFC activity (dominant) and right inferior prefrontal cortex (RIPFC, nondominant) activity (Cabeza et al., 1997; Logan et al., 2002; Stebbins et al., 2002). Increased nondominant activity has been associated with decreased FA values in the anterior corpus callosum (Persson et al., 2006). As such, one might expect healthy young adults with lower anterior callosal FA values to demonstrate greater RIPFC activity during verbal encoding.

Based on the research described above, it is clear that LIPFC activity during encoding predicts subsequent verbal memory, 
however, the impact of any observed RPIFC activity during verbal encoding on subsequent memory in young adults is more difficult to predict. Studies of healthy young adults and splitbrain patients have demonstrated that when the two hemispheres are simultaneously engaged in competing tasks, the performance of each hemisphere declines (Holtzman and Gazzaniga, 1982, 1985; Franz et al., 1996), suggesting that nondominant cortical activity may be detrimental to lateralized task performance. In contrast, additional studies have reported a bilateral processing advantage in young adults as task difficulty increases (Belger and Banich, 1992, 1998; Weissman and Banich, 2000) and some early fMRI studies of verbal encoding in older adults showed that increased RIPFC activity was associated with better subsequent memory (Reuter-Lorenz et al., 2000). However, more recent studies of older adults have challenged these findings (Logan et al., 2002; Persson et al., 2006). Given this conflicting evidence, we predicted that the overall impact of RIPFC activity during verbal encoding on subsequent memory should be negative, but may vary based on individual differences in sex and callosal organization.

\section{Materials and Methods \\ Participants}

Twenty-six participants (15 male; mean age, 18.9 years) were recruited from the Dartmouth community. Data from 14 additional participants were excluded because of technical problems encountered at the time of scanning (13 participants), or corrected recognition performance $<2.5$ SDs below the mean (one participant). All participants were strongly right handed as measured by the Edinburgh Handedness Inventory (Oldfield, 1971). Participants reported no significant abnormal neurological history and all had normal or corrected-to-normal visual acuity. Participants were either paid for their participation or received course credit. All participants gave informed consent in accordance with the guidelines set by the Committee for the Protection of Human Subjects at Dartmouth College.

\section{Experimental task and behavioral data analysis}

Participants performed an incidental verbal encoding task while undergoing fMRI. In two functional runs, participants viewed single words presented on the screen and were asked to determine whether the words were concrete or abstract. 240 words (120 abstract, 120 concrete) were chosen using the MRC Psycholinguistic Database (http://www.psy. uwa.edu.au/mrcdatabase/uwa_mrc.htm). All words were within one SD of mean familiarity (mean, 488; SD, 99). Concrete words were within 1-2 SDs above mean concreteness and abstract words were within 1-2 SDs below mean concreteness (mean, 438; SD, 120). Eighty words (40 abstract, 40 concrete) were presented within each functional run. Words appeared at the center of the screen for $2000 \mathrm{~ms}$. Participants were asked to indicate (via left- and right-handed key presses) whether the word was abstract or concrete. In each run, forty fixation trials were pseudorandomly intermixed with the word trials so that each trial type (concrete, abstract, fixation) followed each other trial type equally often. Fixation trials consisted of a central cross-hair presented for $2500 \mathrm{~ms}$ and introduced "jitter" into the time series so that unique estimates of the hemodynamic responses for the trial types of interest could be computed (Friston et al., 1999).

After the two encoding runs, participants were given a surprise recognition memory test. This took place inside the scanner during the collection of anatomical images. Participants viewed all 160 words that had been presented during functional imaging along with 80 nonstudied new words ( 40 abstract, 40 concrete). Each word was presented individually at the center of the computer screen for $2000 \mathrm{~ms}$. In a two-step recognition memory test, participants were asked to indicate via button press whether or not they remembered the word to be old, and then whether this judgment was with high- or low- confidence. Across participants, the 240 words were counterbalanced between the study and test phases to prevent item effects.
During analysis, participants' responses were divided into four categories: high-confidence hits (correctly responded "old" with high confidence), low-confidence hits, high-confidence misses (incorrectly responded "old" with high confidence), and low-confidence misses. Mean corrected recognition (proportion of hits vs proportion of false alarms) was calculated for each participant.

\section{Neuroimaging parameters and analysis}

All images were acquired using a 1.5T GE Signa scanner (General Electric Medical Systems, Milwaukee, WI) using a standard head coil. All neuroimaging took place at the Dartmouth Brain Imaging Center.

Anatomical imaging. Anatomical images were acquired using a highresolution three-dimensional spoiled gradient recovery sequence [SPGR; 128 sagittal slices; repetition time (TR), $7.7 \mathrm{~ms}$; echo time (TE), $3 \mathrm{~ms}$; flip angle, $15^{\circ}$; voxel size, $1 \times 1 \times 1.2 \mathrm{~mm}$ ]. Using SPM99 (Wellcome Department of Cognitive Neurology, London UK), each individual's highresolution image was normalized to the Montreal Neurological Institute (MNI) template.

Diffusion tensor imaging. Diffusion tensor imaging used a diffusion weighted single-shot spin echo-planar imaging (EPI) sequence. Sixty slices were imaged (slice thickness, $2.5 \mathrm{~mm}$; field of view, $240 \mathrm{~mm}$; matrix size, $128 \times 128)$. For each slice, six diffusion-weighted images $(b=1000$ $\mathrm{s} / \mathrm{mm}^{2}$ ) and 1 reference image $\left(b=0 \mathrm{~s} / \mathrm{mm}^{2}\right.$ ) were acquired (Basser and Pierpaoli, 1998). The sequence was repeated eight times and data were averaged on the scanner. FA images were constructed off-line using Matlab (The MathWorks, Sherborn, MA) scripts implementing formulas described by Basser and Pierpaoli (1996). Using SPM99 (http://www. fil.ion.ucl.ac.uk/spm/software/spm99/), each individual's FA image was coregistered to his or her high-resolution image using a two-step process. The first step coregistered each participant's T2-weighted reference image $(b=0)$ to the mean EPI image from the functional runs, already coregistered to the high-resolution image. The second step coregistered the FA image to this mean EPI image. After coregistration, the FA images were spatially normalized to the International Consortium for Brain Mapping (ICBM) 152 brain template (Montreal Neurological Institute) and smoothed using a $2 \mathrm{~mm}$ full-width at half maximum (FWHM) Gaussian kernel.

For each participant, mean anterior callosal FA was extracted from a 6-mm-diameter sphere located at $(0,29,4)$, the border of the genu and rostrum. This region was chosen because the genu and rostrum connect the prefrontal cortices (de Lacoste et al., 1985; Wakana et al., 2004), including the inferior prefrontal cortical areas of interest. The selection of this region was unbiased by participants' performance or cortical activity levels.

Functional imaging. Functional images were collected in two runs using a gradient spin EPI sequence sensitive to BOLD contrast $\left(\mathrm{T} 2^{*}\right)$ (TR, $2500 \mathrm{~ms}$; TE, $35 \mathrm{~ms}$; flip angle, $90^{\circ} ; 3.75 \times 3.75 \mathrm{~mm}$ in-plane resolution). During each functional run, 124 volumes of axial images ( 25 slices, 4.5 $\mathrm{mm}$ slice thickness, $1 \mathrm{~mm}$ skip between slices) were acquired. Data were processed and analyzed using SPM99. EPI images were corrected for slice timing and motion, coregistered to the individual's high-resolution scan, spatially normalized using the ICBM 152 brain template (Montreal Neurological Institute), and spatially smoothed using a $8 \mathrm{~mm}$ FWHM Gaussian kernel. All coordinates were converted to the standardized coordinate system used by the Talairach Atlas (Talairach and Tournoux, 1988) (http://imaging.mrc-cbu.cam.ac.uk/downloads/MNI2tal/mni2tal.m).

Talairach coordinates are used throughout this paper.

For each participant, general linear models were used to compute parameter estimates $(\beta)$ and $t$-contrast images for each comparison at each voxel. The models combined the two functional runs into one session, included task effects (modeled with a canonical hemodynamic response function and its temporal derivative), the mean for each functional run, a linear trend for each functional run, and motion parameters to model effects of head movement. Included task effects of interest were high-confidence hit trials and miss trials. Miss trials included high and low-confidence misses as well as low-confidence hits. Low-confidence hits were treated as guesses, as participants accurately distinguished between hits and misses when they indicated high confidence (98.9\% correct), but were at chance in distinguishing between hits and misses when 
they indicated low confidence (53.6\% correct). Thus, there was no real difference between lowconfidence hits and low-confidence misses, so low-confidence hits were included with misses.

These individual contrast images were then submitted to a group-level random-effects analysis to create mean $t$ images ( $p<0.001$, uncorrected; minimal cluster size, $\left.5 \mathrm{~mm}^{3}\right)$. An automated search algorithm identified a peak region with the LIPFC associated with word encoding based off a contrast of all trials versus baseline [peak activity centered at $(-53,16,24)$, Brodmann's Area (BA) 44]. No homologous activity within the RIPFC was observed in this group map.

To examine the possibility that individuals with high and low anterior callosal FA values might differ in the degree to which they elicited RIPFC activity, a median split was performed to divide participants into high and low callosal FA groups using each individual's mean FA value extracted from the anterior callosal region of interest. Eleven participants were assigned to the high-FA group (FA $>0.72$; mean FA, 0.77 ; five male; mean age, 18.8 years) and 11 participants were assigned to the low-FA group $(\mathrm{FA}<0.72$; mean FA, 0.62; six males; mean age, 18.6 years). Four participants' FA values were identical to the median and were not assigned to either group. Two independent group-level random effects analyses were then performed to examine activity during word encoding (all vs baseline), one for the high anterior callosal FA group and one for the low anterior callosal FA group ( $p=0.001$, minimal cluster size of five contiguous voxels). Only low anterior callosal FA individuals exhibited activity in the RIPFC [peak activity centered at $(53,15,19)$, BA 44].

Thus, two cortical ROIs were defined from the mean $t$ image comparing all word encoding conditions to baseline; each condition contributed equally to the generation of ROIs. The LIPFC ROI was centered at $(-53$, $16,24)$ and the RIPFC ROI was centered at $(53,15,19)$. These cortical ROIs are located in homologous regions of BA 44 (see Fig. 1).

For all 26 participants, mean parameter estimates within each cortical ROI were then calculated separately for all conditions relative to baseline (all conditions vs baseline), hits relative to baseline (hits vs baseline), and misses relative to baseline (misses vs baseline) using spherical ROIs (8 $\mathrm{mm}$ radius) centered at the peak activity locations. These signal intensities were examined statistically. Specifically, a multiple regression model including the variables of callosal organization, sex, and corrected recognition memory scores was used to predict RIPFC activity during verbal encoding (all conditions vs baseline). Partial correlations were then used to determine the relationship between each predictor variable and RIPFC activity during verbal encoding (all conditions vs baseline) while controlling for the other two predictor variables.

\section{Results \\ Inferior prefrontal cortical activity in the high- and low- callosal organization groups}

Across all participants, LIPFC activity, but not RIPFC activity, was observed during incidental verbal encoding (Fig. 1a). To examine the possibility that individuals with high and low anterior callosal FA might differ in levels of RIPFC activity, a median split based on mean FA within the anterior corpus callosum was performed to divide participants into high and low anterior callosal FA groups (11 participants in each group). To compare BOLD activity during verbal encoding (all conditions vs baseline) between the two hemispheres as a function of callosal organization, a $2 \times 2$ mixed factorial ANOVA [group (high FA, low FA)
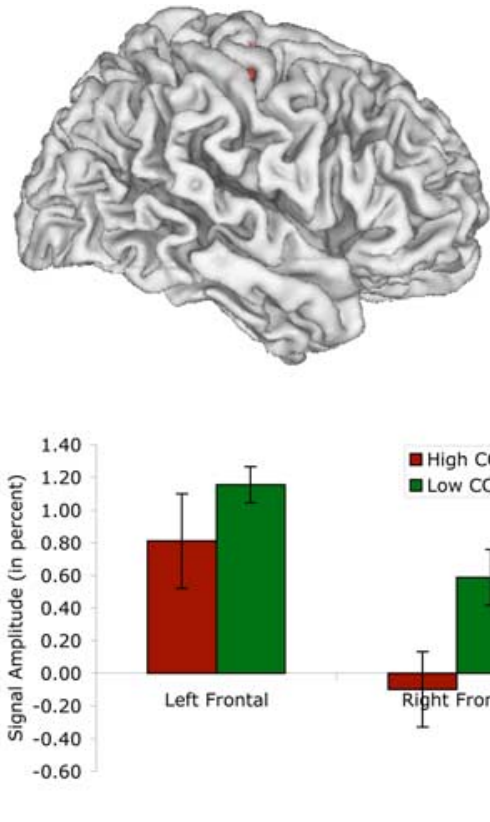

High CC FA - Low CC FA

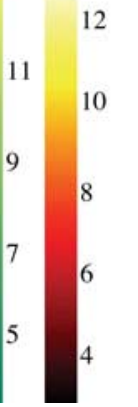

Left Frontal

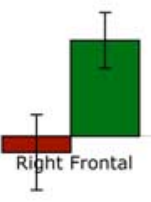

60
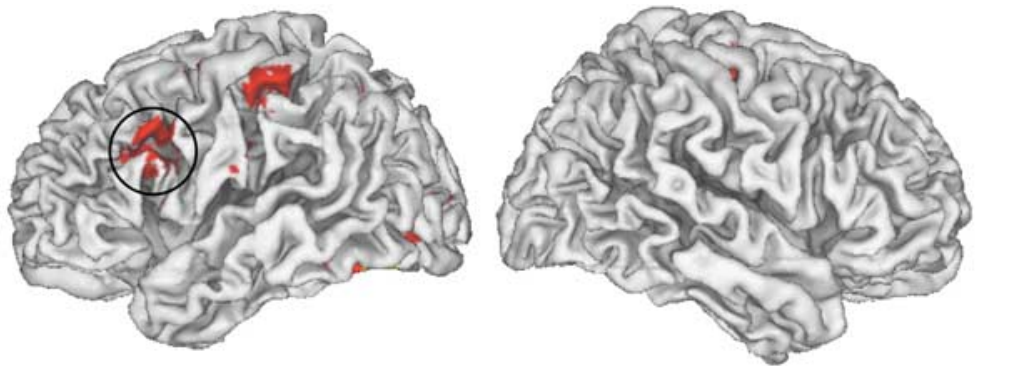

Figure 1. Nondominant cortical activity varies by callosal organization. $\boldsymbol{a}$, Top, During word encoding (all conditions vs aseline), LIPFC activity was observed across all participants. No corresponding RIPFC activity was observed. $\boldsymbol{b}$, Bottom left, ( tude (in percent) during verbal encoding in the $\operatorname{LIPFC}(-53,16,24)$ and $\operatorname{RIPFC}(53,15,19)$ Rols for the high-and low-callosal organization groups. Error bars indicate SEM.

by hemisphere (left, right)] was performed. There was a main effect of hemisphere; both the high and low anterior callosal FA groups demonstrated greater LIPFC activity than RIPFC activity during encoding $\left(F_{(1,20)}=19.8 ; p<0.001\right)$. There was also a main effect of group; the low-FA group exhibited greater cortical activity than the high-FA group regardless of hemisphere $\left(F_{(1,20)}\right.$ $=24.1 ; p<0.001)$. There was no interaction between group and hemisphere $\left(F_{(1,20)}=1.1 ; p=0.32\right)$. Critically, only the low-FA group demonstrated significant RIPFC activity during verbal encoding (mean, 0.59). The high-FA group did not demonstrate significant RIPFC activity during verbal encoding (mean, -0.1 ) $\left(t_{(20)}=2.37 ; p<0.03\right)$ (Figs. $\left.1 b, c\right)$.

To determine whether both the high and low-FA groups demonstrated the expected difference of memory (DM) effect in the LIPFC [greater activity during subsequently remembered words (hits) than during subsequently forgotten words (misses)], a $2 \times$ 2 mixed-factorial ANOVA [group (high FA, low FA) by trial type (hits, misses)] was performed. There was a main effect of trial type; both groups demonstrated greater activity during hits than during misses $\left(F_{(1,20)}=20.1 ; p<0.001\right)$. There was no main effect of group $\left(F_{(1,20)}=1.24 ; p=0.28\right)$ and no interaction between group and trial type $\left(F_{(1,20)}=1.72 ; p=0.20\right)$. Thus, both the high- and low-callosal organization groups demonstrated a significant DM effect within the LIPFC (Fig. 2).

Critically, the high and low anterior callosal FA groups did not differ in overall corrected recognition performance (high-FA group, mean, 0.75 ; low-FA group, mean, $0.79 ; t_{(20)}=1.01 ; p=$ $0.33)$. However, in the high-FA group, RIPFC activity during verbal encoding (all conditions vs baseline) negatively correlated with corrected recognition scores $(r=-0.67 ; p<0.03)$. In the low-FA group, RIPFC activity during verbal encoding (all conditions vs baseline) did not correlate with corrected recognition scores $(r=-0.16 ; p=0.65)$ (Fig. 3$)$. Thus, the impact of RIPFC 

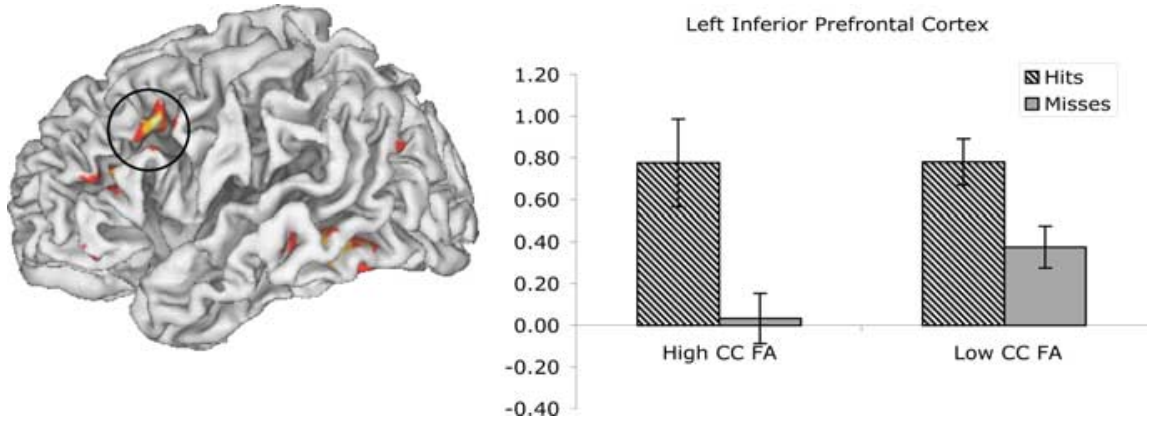

Figure 2. Activity in the LIPFC during encoding of words that were subsequently remembered (hits) and during the encoding of words that were subsequently forgotten (misses). $\boldsymbol{a}$, Left, Across all participants, LIPFC activity was higher during hits than during misses (hits vs misses) ( $p<0.001$ ). $\boldsymbol{b}$, Right, Changes in LIPFC signal amplitude (in percent) during hit trials (hits vs baseline) and miss trials (misses vs baseline) for the high-and low-callosal organization groups. There was a significant difference of memory effect (greater LIPFC activity during hits than during misses) for both groups at a threshold of $p<0.05$. Error bars indicate SEM.

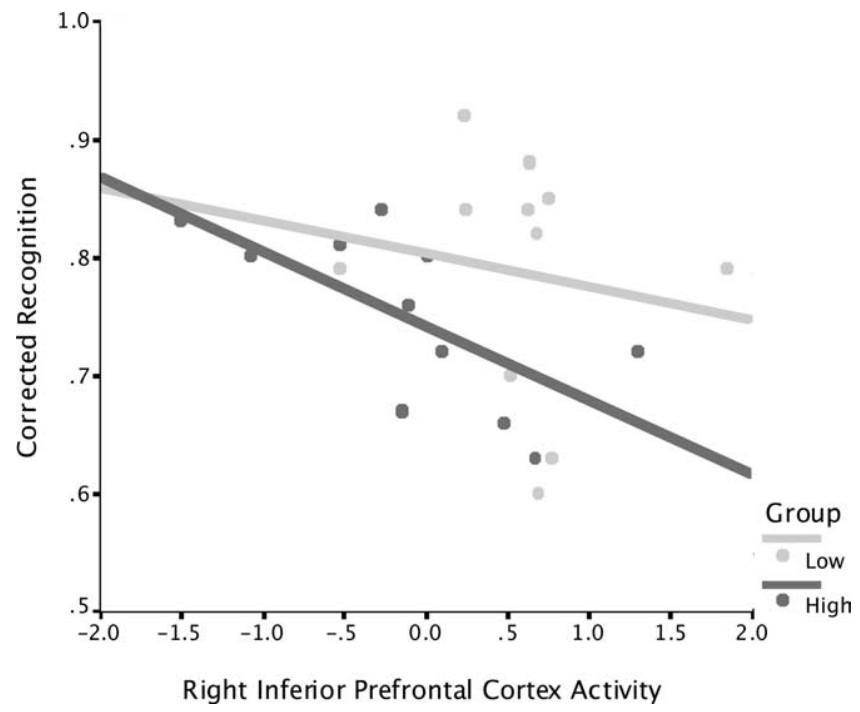

Figure 3. Subsequent verbal memory and RIPFC activity during encoding (all conditions vs baseline) in the high- and low-callosal organization groups. In the high-callosal organization group, greater RIPFC activity during encoding was associated with poorer corrected recognition scores $(p<0.03)$. In the low-callosal organization group, there was no significant correlation between RIPFC activity during encoding and corrected recognition scores $(p=0.65)$.

activity during verbal encoding on subsequent recall depended on the extent of anterior callosal organization (FA).

\section{Predicting nondominant cortical activity across all participants}

A continuous assessment of the data across the entire population of healthy young adults (multiple regression) demonstrated that levels of RIPFC activity during verbal encoding (all conditions vs baseline) were predicted by the variables of anterior callosal FA values, sex, and corrected recognition scores $\left(F_{(3,25)}=4.9 ; p<\right.$ 0.01 , adjusted $R^{2}=0.32$ ). Greater anterior callosal FA was associated with less RIPFC activity $(\beta=-0.54 ; p<0.01)$ (Fig. $4 a$ ). Greater corrected recognition scores were associated with less RIPFC activity ( $\beta=-0.45 ; p<0.02)$ (Fig. $4 b)$. Finally, being male was associated with less RIPFC activity and being female was associated with greater RIPFC activity $(\beta=0.39 ; p<0.05)$, although RIPFC activity in males (mean, 0.04 ) and females (mean, 0.40 ) was not significantly different $\left(t_{(24)}=1.33 ; p=0.2\right)$. Additionally, there were no sex differences in corrected recognition $\left.R^{2}=0.08\right)$ performance $\left(t_{(24)}=0.92 ; p=0.4\right)$ or anterior FA values $\left(t_{(24)}=0.50 ; p=0.6\right)$.

Partial correlations were calculated to determine whether the correlations between RIPFC activity and each predictor variable were significant while controlling for the other two predictor variables. Controlling for sex and corrected recognition performance, anterior callosal FA values negatively correlated with RIPFC activity $(r=-0.55 ; p<0.01)$. Controlling for sex and anterior callosal FA values, corrected recognition performance negatively correlated with RIPFC activity $(r=-0.48$; $p<$ 0.02). Controlling for anterior callosal FA values and corrected recognition performance, sex correlated with RIPFC activity, such that being male was associated with less RIPFC activity and being female was associated with more RIPFC activity $(r=0.44 ; p<0.05)$.

\section{Control analyses}

To test whether anterior callosal FA values, sex, and corrected recognition scores predicted cortical activity in general, anterior callosal FA values, sex, and corrected recognition scores were entered into a multiple regression as predictor variables of LIPFC activity. The regression model did not predict LIPFC activity $\left(F_{(3,25)}=0.45 ; p=0.72\right.$; adjusted $\left.R^{2}=-0.07\right)$.

To test whether anterior callosal FA values specifically predict RIPFC activity in conjunction with sex and corrected recognition scores, a multiple regression was performed with mean FA values from a $6 \mathrm{~mm}$-diameter posterior callosal region [centered at $(0$, $34,17)]$, sex, and corrected recognition scores entered as predictor variables. A posterior callosal region in the splenium was selected to avoid fibers connecting the prefrontal cortices. The regression was not significant $\left(F_{(3,25)}=1.71 ; p=0.19\right.$, adjusted

To test whether differences in RIPFC activity between the high and low anterior callosal FA groups could be accounted for by chance, individuals were randomly assigned to the high- and lowcallosal organization group RIPFC activity levels were compared between the two groups using the $2 \times 2$ mixed-factorial ANOVA [group (high FA, low FA) by hemisphere (left, right)] described above. Across multiple simulations, RIPFC activity varied randomly between the two groups, demonstrating that the observed difference in RIPFC activity between the high and low anterior callosal FA groups was not attributable to chance.

Finally, the results described above used a RIPFC ROI that was defined based on the activity level of the low-callosal organization group. An alternative approach to defining the RIPFC ROI would have been to use the actual anatomical homolog of the LIPFC ROI. This method was not chosen initially because there is growing evidence that the two hemispheres are not entirely symmetric (Keller et al., 2007), and also because this method might have failed to fully capture the nature of cortical activity in the nondominant hemisphere of individuals with low anterior callosal FA. Yet, to ensure that the chosen method did not unduly bias the results, the above analyses were replicated using activity levels in the anatomical RIPFC homolog to the $\operatorname{LIPFC} \operatorname{ROI}(53,16,24)$.

The pattern of cortical activity during verbal encoding (all conditions vs baseline) was the same in both RIPFC ROIs. Activity within the two ROIs did not differ for either the high or low-FA groups (high, $t_{(10)}=0.71, p=0.49$; low, $t_{(10)}=0.19, p=$ 
0.86). Thus, overall, the results were quite similar as those described above using the original RIPFC ROI; however, certain analyses failed to reach statistical significance at $p<0.05$. During verbal encoding (all conditions vs baseline), both the high and low anterior callosal FA groups demonstrated greater LIPFC activity than RPIFC activity $\left(F_{(1,20)}=21.6 ; p<0.001\right)$, and there was no interaction between group (high and low FA) and hemisphere $(\mathrm{LIPFC}, \mathrm{RIPFC})\left(F_{(1,20)}=0.67 ; p=0.42\right)$. Also as described above, RIPFC activity during verbal encoding (all conditions vs baseline) negatively correlated with corrected recognition scores in the high-FA group $(r=-0.63 ; p<0.04)$, and had no impact on corrected recognition scores in the low-FA group $(r=$ $-0.05 ; p=0.89)$. There was a significant trend for only the low-FA group to demonstrate significant RIPFC activity during encoding $\left(t_{(20)}=1.81 ; p<0.09\right)$. Across the entire study population, a multiple regression predicting RIPFC activity using the variables of anterior callosal FA values, sex, and corrected recognition scores also failed to reach statistical significance $\left(F_{(3,25)}=\right.$ $1.68 ; p=0.2)$, however a partial correlation revealed a significant trend for anterior callosal FA values to predict RIPFC activity in the anatomical homolog $(r=-0.38 ; p=0.07)$. Thus, although some of these analyses failed to reach statistical significance, it is clear that when the RIPFC ROI is based on the anatomical, rather than functional, homolog of the LIPFC ROI, the overall pattern of results is the same.

\section{Discussion}

The present study illustrates that that within a population of healthy young adults, individual differences in callosal organization, as measured by fractional anisotropy values, predict the extent and impact of nondominant cortical activity during performance of a lateralized task. Specifically, individuals with lower FA values in the anterior corpus callosum were more likely to demonstrate RIPFC activity during a verbal encoding task. Also, in this subset of individuals, RIPFC activity was not associated with a cost to subsequent verbal memory performance (corrected recognition scores). In contrast, when individuals with greater FA values in the anterior corpus callosum demonstrated RIPFC activity, there was a negative impact on subsequent verbal memory performance. Altogether, these findings suggest that the relative strength of interhemispheric connections is critical in determining the distribution of processing resources between the two hemispheres, and that within a population of healthy young adults, there is significant variation in this relationship.

From a methodological perspective, the use of DTI to characterize individual variation in the anatomical structure of the corpus callosum represents a significant advancement. Historically, the size of the corpus callosum has been used to measure interhemispheric connectivity and shown to be inversely proportional to functional lateralization. Previous studies documented that women and left-handers had larger corpora callosi (DeLacosteUtamsing and Holloway, 1982; Witelson, 1985, 1989; Holloway and de Lacoste, 1986), and were also less lateralized than men and right-handers, particularly with respect to language function (McGlone, 1980; Hecaen et al., 1981; Bryden, 1982; Kimura, 1983). However, callosal size may not reflect the number or type of callosal fibers connecting the two hemispheres (Tomasch,

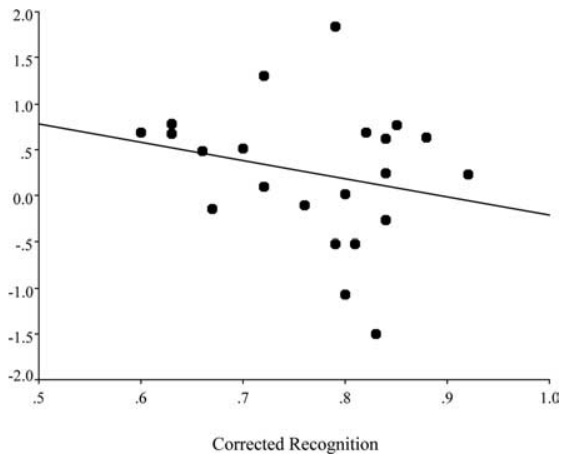
means of quantifying interhemispheric connectivity than callosal size, as FA values depend on the characteristics of the underlying axonal microstructure (e.g., axon size, extent of myelination) (Basser and Jones, 2002; Bozzali et al., 2002; Chepuri et al., 2002; Takahashi et al., 2002; Ciccarelli et al., 2003) that are directly related to the speed of axonal transmission (Waxman and Bennett, 1972).

Combined with measures of cortical activity obtained from fMRI, this more specific means of characterizing the anatomy of the corpus callosum makes it possible to better understand the nature of functional lateralization. If functional lateralization is conceptualized as the extent to which the two hemispheres operate in isolation while performing certain tasks, then the current results suggest that greater fractional anisotropy values through the corpus callosum is associated with increased functional lateralization. In these highly lateralized individuals, nondominant cortical activity may reflect resource competition between homologous cortical regions, which is then detrimental to task performance. In less lateralized individuals, nondominant cortical activity may reflect either the recruitment of additional processing resources of independent intrahemispheric processing, but has no overall impact on task performance.

Several aspects of the current findings support an inhibitory role of callosal fibers in regulating processing resources between the two hemispheres. Inhibitory models of interhemispheric communication predict that greater interhemispheric connectivity enables greater inhibition of the nondominant hemisphere (Kinsbourne, 1970; Cook, 1984). The observed inverse relationship between fractional anisotropy and nondominant cortical activity (increased fractional anisotropy is associated with decreased activity in the nondominant hemisphere) is consistent with this hypothesis. Second, nondominant cortical activity in the high-FA group was associated with a performance cost, suggesting that in this group, greater inhibition of the nondominant hemisphere is required to minimize interference during performance of a lateralized task. Finally, the low-FA group tended to exhibit greater cortical activity in both hemispheres (although this was not significant in the dominant hemisphere), suggesting that relatively weaker interhemispheric connectivity is associated with reduced reciprocal inhibition of the two hemispheres. However, we would like to emphasize that the present study was not designed to explicitly test whether the nature of callosal interactions is inhibitory or excitatory, and it certainly seems reasonable to assume that both types of communication exist in the intact human brain. Indeed, as noted in the introduction, there is evi- 
dence to suggest that the impact of nondominant cortical activity on the performance of a lateralized task may depend on task demands; under some circumstances, bilateral activity is associated with performance decline (Holtzman and Gazzaniga, 1982, 1985; Franz et al., 1996), and under different circumstances, bilateral cortical activity is associated with performance enhancement (Belger and Banich, 1992, 1998; Weissman and Banich, 2000). Furthermore, one might expect that successful inhibition of the nondominant hemisphere might be associated with greater activity in the dominant hemisphere, and this pattern was not observed. Thus, the current study is limited in its ability to dissociate between the inhibition and excitation views of callosal interaction, but strongly suggests that the strength of callosal connections determines the effectiveness of interhemispheric communication. Furthermore, as described above, previous research suggests that task demands may determine whether callosal connections mediate inhibition or excitation of the nondominant hemisphere.

Related to this, it is also possible that the nature of interhemispheric communication varies depending on the cortical areas that are involved in performing the task. To date, most studies examining individual differences in patterns of laterality, including the current study, have tended to use tasks for which there is a well established pattern of hemispheric asymmetry. Such tasks primarily elicit differences in cortical activity levels of the prefrontal, and to a lesser extent, medial temporal cortices (Kelley et al., 1998; Wagner et al., 1998a). Although this may partially reflect the serendipitous course of research, it does seem that particular areas of the brain, including the frontal lobes, are more likely to exhibit lateralized functions. This may be related to the unique ontology and phylogeny of the frontal lobes, and the anterior corpus callosum connecting them.

Finally, as also mentioned in the introduction, the frontal lobes and the anterior corpus callosum seem to be particularly vulnerable to age-related changes (Raz et al., 1997; Resnick et al., 2003; Head et al., 2004), suggesting that callosally mediated dynamics between the two hemispheres shift across the lifespan. Consistent with this, a series of studies has demonstrated that hemispheric asymmetries decline with age (Cabeza et al., 1997; Logan et al., 2002; Stebbins et al., 2002), and that increased nondominant cortical activity is associated with decreased fractional anisotropy in the anterior corpus callosum (Persson et al., 2006). The current results suggest that the strength of interhemispheric connectivity, and the associated activity in the nondominant hemisphere during performance of a lateralized task, varies even within a population of healthy young adults. This finding raises the possibility that in some older adults, the pattern of reduced functional lateralization and reduced fractional anisotropy in the corpus callosum represents a stable state across adulthood rather than age-related changes. Thus, when interpreting age-related changes in functional lateralization, the possibility of longstanding individual differences in the strength of callosal connections and cortical activity should be addressed.

In summary, the present study combines two neuroimaging techniques, fMRI and DTI, to paint a complex picture of the nature of hemispheric asymmetries in the intact human brain. During verbal encoding, greater fractional anisotropy in the anterior corpus callosum was associated with less RIPFC activity, suggesting that under these task conditions, the corpus callosum may mediate inhibition of nondominant cortical activity. Furthermore, the anatomical organization of the corpus callosum, as measured by fractional anisotropy, predicted the impact of nondominant cortical activity on task performance, suggesting that the strength of interhemispheric connectivity and functional lateralization determines behavioral performance. Future research is needed to determine whether this relationship holds across task conditions and the developmental lifespan, but the current research strongly suggests that individual differences in the relationship between the anatomical organization of the corpus callosum and associated patterns of cortical activity should integrated into this research.

\section{References}

Aboitiz F, Scheibel AB, Zaidel E (1992a) Morphometry of the human corpus callosum, with emphasis on sex differences. Brain 115:1521-1541.

Aboitiz F, Scheibel AB, Fisher RS, Zaidel E (1992b) Fiber composition of the human corpus callosum. Brain Res 598:143-153.

Basser PJ, Jones DK (2002) Diffusion-tensor MRI: theory, experimental design and data analysis-a technical review. NMR Biomedicine 15:456-467.

Basser PJ, Pierpaoli C (1996) Microstructural and physiological features of tissues elucidated by quantitative-diffusion-tensor MRI. J Mag Res B 111: 209-219.

Basser PJ, Pierpaoli C (1998) A simplified method to measure the diffusion tensor from seven MR images. Magn Reson Med 39:928-934.

Belger A, Banich MT (1992) Interhemispheric interaction affected by computational complexity. Neuropsychologia 31:923-929.

Belger A, Banich MT (1998) Costs and benefits of integrating information between the cerebral hemispheres: a computational perspective. Neuropsychology 12:380-398.

Bozzali M, Falini A, Franceschi M, Cercignani M, Zuffi M, Scotti G, Comi G, Filippi M (2002) White matter damage in Alzheimer's disease assessed in vivo using diffusion tensor magnetic resonance imaging. J Neurol Neurosurg Psychiatry 72:742-746.

Bryden MP (1982) Laterality: functional asymmetry in the intact brain. New York: Academic.

Cabeza R (2002) Hemispheric asymmetry reduction in older adults: the HAROLD model. Psychol Aging 17:85-100.

Cabeza R, Grady CL, Nyberg L, McIntosh AR, Tulving E, Kapur S, Jennings JM, Houle S, Craik FI (1997) Age-related differences in neural activity during memory encoding and retrieval: A positron emission tomography study. J Neurosci 17:391-400.

Chepuri NB, Yen Y-F, Burdette JH, Li H, Moody DM, Maldjian JA (2002) Diffusion anisotropy in the corpus callosum. Am J Neuroradiol 23:803-808.

Ciccarelli O, Werring DJ, Barker GJ, Griffin CM, Wheeler-Kingshott CA, Miller DH, Thompson AJ (2003) A study of the mechanisms of normalappearing white matter damage in multiple sclerosis using diffusion tensor imaging: evidence for Wallerian degeneration. J Neurology 250:287-292.

Cook ND (1984) Homotopic callosal inhibition. Brain Lang 23:116-125.

de Lacoste MC, Kirkpatrick JB, Ross ED (1985) Topography of the human corpus callosum. J Neuropathol Exp Neurol 44:578-591.

DeLacoste-Utamsing C, Holloway RL (1982) Sexual dimorphism in the human corpus callosum. Science 216:1431-1432.

Franz E, Ivry R, Gazzaniga MS (1996) Dissociation of spatial and temporal coupling in the bimanual movements of callosotomy patients. Psychol Sci 7:306-310.

Friston KJ, Zarahn E, Josephs O, Henson RN, Dale AM (1999) Stochastic designs in event-related fMRI. NeuroImage 10:607-619.

Gazzaniga MS (2000) Cerebral specialization and interhemispheric communication: Does the corpus callosum enable the human condition. Brain 123:1293-1326.

Head D, Buckner RL, Shimony JS, Williams LE, Akbudak E, Conturo TE, McAvoy M, Morris JC, Snyder AZ (2004) Differential vulnerability of anterior white matter in nondemented aging with minimal acceleration in dementia of the Alzheimer type: Evidence from diffusion tensor imaging. Cerebral Cortex 14:410-423.

Hecaen HM, DeAgostini M, Monzon-Montes A (1981) Cerebral organization in left-handers. Brain and Language 12:261-284.

Holloway RL, de Lacoste MC (1986) Sexual dimorphism in the human corpus callosum: An extension and replication study. Hum Neurobiol 5:87-91. 
Holtzman JD, Gazzaniga MS (1982) Dual task interactions due exclusively to limits in processing resources. Science 218:1325-1327.

Holtzman JD, Gazzaniga MS (1985) Enhanced dual task performance following corpus commissurotomy in humans. Neuropsychologia 23:315-321.

Keller SS, Highley JR, Garcia-Finana M, Sluming V, Rezaie R, Roberts N (2007) Sulcal variability, stereological measurement and asymmetry of Broca's area on MR images. J Anat 211:534-555.

Kelley WM, Miezin FM, McDermott KB, Buckner RL, Raichle ME, Cohen NJ, Ollinger JM, Akbudak E, Conturo TE, Snyder AZ, Petersen SE (1998) Hemispheric specialization in human dorsal frontal cortex and medial temporal lobe for verbal and nonverbal memory encoding. Neuron 20:927-936.

Kimura D (1983) Speech representation in an unbiased sample of lefthanders. Hum Neurobiol 2:147-154.

Kinsbourne M (1970) A model for the mechanism of unilateral neglect of space. Trans Am Neurol Assoc 95:143-146.

Logan JM, Sanders AL, Snyder AZ, Morris JC, Buckner RL (2002) Underrecruitment and nonselective recruitment: dissociable neural mechanisms associated with aging. Neuron 33:827-840.

McGlone J (1980) Sex differences in human brain asymmetry: a critical survey. Behav Brain Sci 3:215-263.

Oldfield RC (1971) The assessment and analysis of handedness: the Edinburgh inventory. Neuropsychologia 9:97-113.

Persson J, Nyberg L, Lind J, Larsson A, Nilsson LG, Ingvar M, Buckner RL (2006) Structure-function correlates of cognitive decline in aging. Cereb Cortex 16:907-915.

Raz N, Gunning FM, Head D, Dupuis JH, McQuain J, Briggs SD, Loken WJ, Thornton AE, Acker AD (1997) Selective aging of the human cerebral cortex observed in vivo: differential vulnerability of the prefrontal gray matter. Cereb Cortex 7:268-282.

Resnick SM, Pham DL, Kraut MA, Zonderman AB, Davatzikos C (2003) Longitudinal magnetic resonance imaging studies in older adults: a shrinking brain. J Neurosci 23:3295-3301.

Reuter-Lorenz PA, Jonides J, Smith EE, Hartley A, Miller A, Marshuetz C, Koeppe RA (2000) Age differences in the frontal lateralization of verbal and spatial working memory revealed by PET. J Cogn Neurosci 12:174-187.

Stebbins GT, Carrillo MC, Dorfman J, Dirksen C, Desmond JE, Turner DA, Bennett DA, Wilson RS, Glover G, Gabrieli JD (2002) Aging effects on memory encoding in the frontal lobes. Psychol Aging 17:44-55.

Takahashi S, Yonezawa H, Takahashi J, Kudo M, Inoue T, Tohgi H (2002) Selective reduction of diffusion anisotropy in white matter of Alzheimer disease brains measured by 3.0 Tesla magnetic resonance imaging. Neurosci Lett 332:45-48.

Talairach J, Tournoux P (1988) Co-planar stereotaxic atlas of the human brain. Stuttgart, Germany: Georg Thieme Verlag.

Tomasch J (1954) Size, distribution, and number of fibres in the human corpus callosum. Anat Rec 119:119-135.

Wagner AD, Poldrack RA, Eldridge LL, Desmond JE, Glover GH, Gabrieli JD (1998a) Material-specific lateralization of prefrontal activation during episodic encoding and retrieval. NeuroReport 9:3711-3717.

Wagner AD, Schacter DL, Rotte M, Koutstaal W, Maril A, Dale AM, Rosen BR, Buckner RL (1998b) Building memories: remembering and forgetting of verbal experiences as predicted by brain activity. Science 281:1188-1191.

Wakana S, Jiang H, Nagae-Poetscher LM, vanZijl PC, Mori S (2004) Fiber tract-based atlas of human white matter anatomy. Radiology 230:77-87.

Waxman SG, Bennett MVL (1972) Relative conduction velocities of small myelinated and non-myelinated fibres in the central nervous system. Nat New Biol 238:217-219.

Weissman DH, Banich MT (2000) The cerebral hemispheres cooperate to perform complex but not simple tasks. Neuropsychology 14:41-59.

Wig GS, Miller MB, Kingstone A, Kelley WM (2004) Separable routes to human memory formation: dissociating task and material contributions in prefrontal cortex. J Cogn Neurosci 16:139-148.

Witelson SF (1985) The brain connection: the corpus callosum is larger in left-handers. Science 229:665-668.

Witelson SF (1989) Hand and sex differences in the isthmus and genu of the human corpus callosum. Brain 112:799-835.

Witelson SF, Goldsmith CH (1991) The relationship of hand preference to anatomy of the corpus callosum in men. Brain Res 545:175-182. 